\title{
The correspondence principle and the understanding of decoherence
}

Sebastian Fortin and Olimpia Lombardi

CONICET and University of Buenos Aires

Corresponding author: Olimpia Lombardi, olimpiafilo@gmail.com

\begin{abstract}
Although Bohr's Correspondence Principle (CP) played a central role in the first versions of quantum mechanics, its original version seems to have no present-day relevance. The purpose of the present article is to show that the $\mathrm{CP}$, with no need of being interpreted in terms of the quantum-to-classical limit, still plays a relevant role in the understanding of the relationships between the classical and the quantum domains. In particular, it will be argued that a generic version of the $\mathrm{CP}$ is very helpful for elucidating the physical meaning of the phenomenon of quantum decoherence.
\end{abstract}




\title{
The correspondence principle and the understanding of decoherence
}

\author{
Sebastian Fortin and Olimpia Lombardi
}

CONICET and University of Buenos Aires

\section{1.- Introduction}

Although historians and philosophers of physics agree on that Niels Bohr's Correspondence Principle $(\mathrm{CP})$ played a central role in the first versions of quantum mechanics, in general they do not reach a consensus about the interpretation of the principle. Nevertheless, independently of this disagreement, a good question is what relevance the principle may have at present, since it was formulated in the context of the "old quantum theory," already set aside in the present-day practice of physics. Perhaps precisely for this reason most physicists rapidly forgot the original presentation of the $\mathrm{CP}$, and the principle turned out to be conceived as the general requirement that quantum mechanics approximate the results of classical mechanics in the appropriate limit. As a consequence, the CP in its Bohr's original version became only interesting for the historians of quantum mechanics.

The purpose of the present article is to recover Bohr's original version of the CP as a useful theoretical tool to face conceptual problems in the foundations of quantum mechanics. From this perspective, we will show that the $\mathrm{CP}$, with no need of being interpreted in terms of a quantum-to-classical limit, still plays a relevant role in the understanding of the relationships between the classical and the quantum domains. In particular, it will be argued that a generalized version of the original $\mathrm{CP}$ is very helpful for elucidating the physical meaning of the phenomenon of quantum decoherence. For this purpose, in Section 2, the origins of the $\mathrm{CP}$ in the old quantum theory will be recalled, stressing how a claim about the relationship between frequencies and motions in the model of the atom gradually became a requirement about the classical limit of quantum mechanics. Section 3 will deal with Robert Batterman's proposal of appealing to the CP, in its original formulation, as a conceptual tool to face the hard problem of defining quantum chaos. Batterman's strategy will lead us in the way toward the elucidation of the concept of decoherence. In order to follow this way, first the theoretical foundations of quantum decoherence will be summarized in Section 4, stressing the issue of the interpretation of the reduced state in terms of what Bernard d'Espagnat called "improper mixture". Section 5 will be devoted to elucidate the nature of the reduced state by establishing the analogy between the classical and the quantum cases. Such an analogy, manifested as a structural correspondence at the level of expectation values, shows that quantum reduced states are coarse descriptions, analogously to coarse-grained 
states and to reduced states in classical statistical mechanics. This result will be the basis, in Section 6, to generalize the $\mathrm{CP}$ as the correspondence between analogous quantum and classical concepts and regularities, and to conceive the structural correspondence presented in the previous section as a particular case of this generalized CP. Thus, following Batterman's strategy regarding using the $\mathrm{CP}$ as a tool to face conceptual difficulties in quantum mechanics, it can be concluded that, since quantum reduced states are coarse descriptions, then decoherence is a coarse phenomenon, relative to the relevant observables considered for the description. Finally, in the last section, after summarizing the main results of the article, the conceptual lines opened by this way of conceiving decoherence will be pointed out, in particular those related to the classical limit of quantum mechanics and to quantum chaos.

\section{2.- From the correspondence principle to the classical limit}

As Alisa Bokulich (2014) points out, the CP was not only an essential assumption that marked the birth of quantum mechanics, but also the cornerstone of Bohr's interpretation of the theory, strongly linked to his view of complementarity. In a nutshell, the principle establishes a correspondence between radiation and motion: the energy spectrum of an atom, with the possible transitions between stationary states, is connected with the periodic motions of the electrons in the atom.

Although in Bohr's 1913 lecture "On the constitution of molecules and atoms" the idea of correspondence is preannounced, it is in the 1918 article "On the quantum theory of line spectra" that Bohr explicitly talks about "the analogy between the quantum theory and the ordinary theory of radiation" (Bohr 1918: 70). The terms 'correspondence' and 'correspondence principle' appear for the first time in a lecture of 1920, in which Bohr explains that, despite the difference between ordinary electrodynamics and the new theory,

"there is found, nevertheless, to exist a far-reaching correspondence between the various types of possible transitions between the stationary states on the one hand and the various harmonic components of the motion on the other hand." (Bohr 1920, in Bohr 1976: 245-246).

As Robert Batterman (1991) stresses, in these first works, the CP does not amount to a claim to the effect that the quantum theory must contain the classical theory as a limiting case. By contrast, Bohr claims that

"[t]his application of the Correspondence Principle [...] on the whole expresses clearly the close connection between radiation and motion in the quantum theory, 
which persists in spite of the fundamental difference between the character of the postulates and the continuous description of the classical theory." (Bohr 1924: 26)

In other words, Bohr does not conceive the $\mathrm{CP}$ as stating the agreement between the classical and the quantum descriptions in a limiting domain, but rather, as a substantive claim: "this Correspondence Principle must be regarded purely as a law of the quantum theory" (Bohr 1924: 22). Thus, in Bohr's version, the CP is first formulated as a contingent claim derived from experimental facts, but then it is promoted to the status of a principle, that is, of a law of the theory, endowed with physical necessity.

Some contemporary physicists embraced Bohr's CP and even developed it further. This is the case of Hendrik Kramers in his 1919 doctoral dissertation under Bohr supervision, and of John van Vleck in a 1924 article, which bears the subtitle "Some extensions of the correspondence principle." Nevertheless, and despite Bohr's high reputation, several contemporary authors manifested their dissatisfaction regarding the CP: if quantum mechanics is a fundamental theory, in principle universally applicable, it is difficult to understand why merely "phenomenological" classical facts play a relevant role in the understanding of the quantum domain. This was the view of Arnold Sommerfeld, who considered the $\mathrm{CP}$ "a magic wand [...], which allows us immediately to make use of the results of the classical wave theory in the quantum theory." (Sommereld 1923: 275). Sommerfeld's dislike of CP seems to be the result of what he considered an illegitimate mixing of quantum and classical concepts: "I cannot view it [the $\mathrm{CP}$ ] as ultimately satisfying on account of its mixing of quantum-theoretical and classical viewpoints. (Sommerfeld 1924: 1048).

Perhaps due to the influence from Sommerfeld, who was the supervisor of his doctoral dissertation, Wolfgang Pauli was also very skeptical about the relevance of the CP. In fact, he outrightly rejected Bohr's suggestion of grounding the exclusion principle on CP:

"I personally do not believe, however, that the correspondence principle will lead to a foundation of the rule [...].The justification of the exclusion [...] in the $\mathrm{H}$ atom by pointing to the collision with the nucleus has never pleased me much. It would be much more satisfying if we could understand directly on the grounds of a more general quantum mechanics (one that deviates from classical mechanics)."

(Pauli to Bohr, December 31st, 1924, quoted in Heilbron 1983: 306)

This dissatisfaction with the $\mathrm{CP}$ seems to contrast with the fact that, in his doctoral dissertation, Pauli (1922) had introduced what he had called a "mechanical correspondence 
principle", in analogy with the original electrodynamical CP as proposed by Bohr. Pauli's idea was that classical mechanics should break down when applied to inelastic collision processes because the amount of energy transferred in the process cannot be continuous. However, the seed of a reinterpretation of the CP can be found already in this idea, since Pauli's initial assumption was the requirement that the results of quantum theory and classical mechanics coincide in the case of large quantum numbers, where the energy states converge toward a continuum. As Robert Rynasiewicz points out, the advance toward a reinterpretation of the CP was perceived by some contemporary physicists, who complained that "Pauli did not establish a correspondence, in Bohr's sense of the word” (Rynasiewicz 2015: 184).

Pauli's treatment of the CP was the first step toward a new reading of the principle in terms of the classical limit of quantum mechanics. In fact, just a few years after Pauli's dissertation, Paul Dirac characterized the $\mathrm{CP}$ as the requirement that "the classical theory gives the right results in the limiting case when the action per cycle of the system is large compared to Planck's constant $h$, and in certain other special cases." (Dirac 1925: 642). Since then, the CP began to be conceived as a limiting requirement, and under this reading appeared in the most used textbooks of quantum mechanics. For instance, in Max Born's classic book, the $\mathrm{CP}$ is introduced with the following words:

“The leading idea (Bohr's correspondence principle, 1923) may be stated broadly as follows. Judged by the test of experience, the laws of classical physics have brilliantly justified themselves in all processes of motion [...] it must be demanded that, for the limiting cases of large masses and of orbits of large dimensions, the new mechanics passes over into classical mechanics." (Born 1933: 103)

The CP is introduced in David Bohm's well-known textbook in a similar way:

"[T]he correspondence principle [...] states that the laws of quantum physics must be so chosen that in the classical limit, where many quanta are involved, the quantum laws lead to the classical equations as an average" (Bohm 1951:31)

Summing up, from being a principle that establishes a correspondence between radiation and motion in Bohr's writings, the $\mathrm{CP}$ gradually became the general requirement that quantum mechanics be able to recover the empirical success of classical mechanics in the limit of large masses and orbits of large dimensions. It may seem that the classical limit requirement is the only version of the $\mathrm{CP}$ that makes sense to survive in the standard version of quantum mechanics, once the old quantum theory has been set aside in the current practice 
of physics. However, here we will focus on Bohr's original "correspondence" version, in order to show that it can still be useful for the conceptual understanding of certain aspects of the theory.

\section{3.- Correspondence principle and quantum chaos}

Rynasiewicz (2015) explicitly points out the tension "constraint versus postulate" regarding the interpretation of the $\mathrm{CP}$, that is, the tension between viewing the $\mathrm{CP}$ as a heuristic principle for theory construction and conceiving it as a law of quantum mechanics. Perhaps the two views are not incompatible: even if for Bohr himself the CP was a fundamental law of quantum mechanics, retrospectively it may be interpreted as a leading heuristic factor in the construction of the theory. Anyway, at present the CP seems to be superfluous from the two viewpoints: it is clearly not a part of the new formalism, nor is it necessary to construct a theory that is already complete. Despite this unfavorable opinion regarding the present-day relevance of the $\mathrm{CP}$, here it will be shown that the principle can still play an interesting role in the understanding of the relationship between the quantum and the classical domains. Let us see this in the case of quantum chaos.

Although the existence of chaos in classical mechanics has been rigorously proved only in a few highly idealized systems, the behavior of many classical systems exhibits features that can be easily interpreted as results emerging from a chaotic dynamics. These empirical manifestations contrast with the fact that chaos in quantum systems seems to be the exception rather than the rule. The relative scarcity of quantum chaos has been repeatedly conceived as a challenge for the $\mathrm{CP}$ : "some have used the quantum chaos problem to cast doubt on the correspondence principle and, as a result, on the empirical adequacy of quantum mechanics" (Kronz 1998: 51). For instance, Belot and Earman talk about the "widely shared sense that chaos presents a challenge either to quantum mechanics itself or to our understanding of the correspondence principle" (Belot and Earman 1997: 149).

At least two general strategies have been adopted to stress the pressure that chaos seems to put on the CP. The first one is a top-down strategy, which relies on quantizing simple classical chaotic models: the problem is that the resulting quantum models are usually nonchaotic according to some feature considered as an indicator of chaos. This is the argumentative path followed by Joseph Ford and his colleagues (Ford, Mantica, and Ristow 1991, Ford and Mantica 1992): by taking the notion of complexity as the key concept for defining chaos, they argue that the systems resulting from the quantization of classical chaotic systems have null complexity and, therefore, are intrinsically non-chaotic. On this basis, the 
authors conclude that the $\mathrm{CP}$ has been refuted and that quantum mechanics should be replaced with a theory capable of accounting for chaotic behavior.

The second general strategy consists in searching for the usual indicators of chaos directly in quantum systems and verifying that those indicators are absent in quantum evolutions. The particular arguments differ from each other with respect to the specific feature to be regarded as the relevant indicator of chaotic behavior. For instance, when the exponential divergence of trajectories is focused, the usual claim is that quantum mechanics suppresses chaos because it is not possible to define precise trajectories in quantum evolutions (see, e.g., Schuster 1984). Since non-linearity is usually taken as a necessary condition for chaos in classical systems, some researchers have concluded that quantum systems are unavoidably non-chaotic because the Schrödinger equation is linear (Berry 1989). Another feature that has been used to explain the scarcity of quantum chaos is the unitarity of the Schrödinger evolutions: due to the fact that quantum evolutions change neither the angle nor the distance between vectors corresponding to different states, quantum systems are not sensitive to initial conditions and, therefore, they are non-chaotic (see discussion in Kronz 1998).

Independently of how the $\mathrm{CP}$ is conceived, the different views about the tension between chaos and quantum mechanics disagree on which the correct or, at least, the best indicator of quantum chaos is. This disagreement points to a problem that is conceptually previous to the supposed tension: what 'chaos at the quantum level' means. Since the concept of chaos still lacks a precise and completely general definition in the classical domain (for a detailed conceptual discussion, see Batterman 1993; for the difference among mathematical definitions, see Smith 1998: Chapter 10), drawing fatal conclusions from a supposed scarcity of quantum chaos seems to be a too extreme maneuver. Perhaps for this reason Batterman turns the problem upside down: the issue is not to understand why there is no chaos in the quantum domain, but rather to understand what quantum chaos means. And in the search for a definition of quantum chaos, the $\mathrm{CP}$ plays a guiding role:

"(2) the CP can be maintained and, in fact, used to further investigate the connections between classical motions and quantum properties and processes. If (2) holds, then it may be quite reasonable to think that the CP can serve as a guide or signpost to finding a proper definition of quantum chaos." (Batterman 1991: 215) 
But, as remarked in the previous section, Batterman stresses that Bohr does not understand the $\mathrm{CP}$ as simply expressing that the two theories must agree in a limiting domain. By contrast, the $\mathrm{CP}$ is a substantive claim that expresses an intimate connection between periodic classical motion and the stationary states of quantum systems.

Batterman's strategy consists in extending the method that he calls 'torus quantisation' (following Berry 1983) to the case of systems whose classical counterparts may exhibit chaos. Torus quantization, strictly valid in the integrable case, shows that the classical periodic orbits can be used to yield the quantum energy spectrum. Batterman finds the modern expression of the $\mathrm{CP}$ in the extension of the correspondence between periodic motion and energy spectrum to the ergodic case. On the basis of this reading, "the CP [...] makes plausible a definition of quantum chaos in terms of the statistics of spectra." (Batterman 1991: 221). As a consequence, Batterman concludes that "once the CP is properly interpreted, it is not threatened by the fact that QM does not appear to exhibit chaotic behavior of the kind present in CM. In fact, [...] the CP actually does help settle the definitional debate." (Batterman 1991: 191).

In the present context the interest is focused not on the technical details of Batterman's proposal, but rather on his general strategy: given a conceptual problem in the quantum domain, in particular regarding its relationship with the classical world, the CP is appealed to as a guiding principle that provides the framework for a satisfactory solution. The plan is to apply the same strategy to face certain conceptual problems related to the interpretation of quantum decoherence. For this purpose, the theoretical foundations of decoherence and certain conceptual difficulties of the decoherence program will be first recalled.

\section{4.- Environment-induced decoherence}

The roots of the environment-induced decoherence program can be found in certain works of the seventies, in which the measurement problem began to be addressed from an open-system perspective: the point was that macroscopic systems, such as measurement apparatuses, are never perfectly closed, but interact significantly with their environments (see Zeh 1970, 1973). On the basis of those pioneers works, the theory of decoherence was systematized and developed by Wojciech Zurek (1981, 1982, 1991, 2003) and his collaborators, by conceiving decoherence as a process resulting from the interaction between a quantum system and its environment. Let us recall the basics of this environment-induced decoherence approach, at present the orthodox approach to quantum decoherence. 
Given an open quantum system $S$ in the state $\sum_{i} c_{i}\left|\varphi_{i}\right\rangle$ and its environment $E$, the initial state of the whole system $S E$ can be expressed as

$$
|\Psi(0)\rangle=\sum_{i} c_{i}\left|\varphi_{i}\right\rangle \otimes\left|\varepsilon_{0}\right\rangle
$$

where $\left|\varepsilon_{0}\right\rangle$ is the state of the environment before its interaction with $S$. The initial state $|\Psi(0)\rangle$ evolves into $|\Psi(t)\rangle$, such that the density operator $\hat{\rho}(t)=|\Psi(t)\rangle\langle\Psi(t)|$ results

$$
\hat{\rho}(t)=\sum_{i j} c_{i} c_{j}^{*}\left|\varphi_{i}\right\rangle\left|\varepsilon_{i}(t)\right\rangle\left\langle\varphi_{j}\right|\left\langle\varepsilon_{j}(t)\right|
$$

where the $\left|\varepsilon_{i}(t)\right\rangle$ are the states of the environment associated with the different states $\left|\varphi_{i}\right\rangle$, and the $c_{i} c_{j}^{*} \neq 0$, with $i \neq j$, represent the quantum correlations that preclude classicality. The orthodox approach relies on computing the reduced density operator $\hat{\rho}_{r}^{S}(t)$ of the open system $S$, which results from tracing over the environmental degrees of freedom:

$$
\hat{\rho}_{r}^{S}(t)=\operatorname{Tr}_{E} \hat{\rho}(t)=\sum_{i j} c_{i} c_{j}^{*}\left|\varphi_{i}\right\rangle\left\langle\varphi_{j}\right|\left\langle\varepsilon_{i}(t) \mid \varepsilon_{j}(t)\right\rangle
$$

where the factor $\left\langle\varepsilon_{i}(t) \mid \varepsilon_{j}(t)\right\rangle$, with $i \neq j$, determines the size of the off-diagonal terms at each time. Many standard models for the interaction Hamiltonian show that, for environments with a very large number of degrees of freedom, the states $\left|\varepsilon_{i}\right\rangle$ rapidly approach orthogonality, $\left\langle\varepsilon_{i}(t) \mid \varepsilon_{j}(t)\right\rangle \rightarrow \delta_{i j}$. As a consequence, after an extremely short "decoherence time", the reduced state of the open system becomes diagonal in a "pointer basis" $\left\{\left|\varphi_{i}\right\rangle\right\}$ corresponding to the classically behaving observable:

$$
\hat{\rho}_{r}^{S}(t) \longrightarrow \hat{\rho}_{r}^{S}=\sum_{i}\left|c_{i}\right|^{2}\left|\varphi_{i}\right\rangle\left\langle\varphi_{i}\right|
$$

According to the orthodox interpretation of decoherence, the decohered state $\hat{\rho}_{r}^{S}$ is the quantum state of the open system $S$ and represents a quantum mixture that contains only the terms corresponding to classical correlations. It is precisely for this reason that decoherence would offer the essential ingredient for solving the quantum measurement problem and for explaining the quantum-to-classical transition. In particular, Zurek conceives the process of decoherence as the dynamical description of the phenomenon of collapse (see Zurek 1981), since "quantum entanglement will be converted into an effectively classical correlation as a result of the interaction" (Paz and Zurek 2002: 90). As a consequence, decoherence would supply the explanation of the emergence of classicality: "the environment distills the classical essence of a quantum system." (Zurek 2003: 3). 
This way of conceiving the physical phenomenon led Zurek and his collaborators to claim that decoherence produces the "degradation" of pure states into mixtures:

"When the system is open, interaction with the environment will inevitably result in an incessant "monitoring" of some of the observables by the environmental degrees of freedom. This will result in the degradation of the pure states into mixtures." (Paz and Zurek 2002: 14)

"pure states turn into mixtures and rapidly diagonalize in the einselected states" (Zurek 2003: 13)

In order to quantify such a "degradation", the evolution of the purity $\zeta$ of the open system is studied, as defined as (see Zurek 2003: 18):

$$
\zeta(t)=\operatorname{Tr}\left(\hat{\rho}_{r}^{S}(t)\right)^{2}
$$

According to Zurek, this quantity is equal to one for a pure state and decreases when the state becomes mixed.

However, Bernard d'Espagnat (1976) warned against the interpretation of reduced states as quantum states by introducing the already classical distinction between proper and improper mixtures: whereas a proper mixture is the quantum state of a closed system, an improper mixture is a density operator obtained by disregarding some degrees of freedom of the closed system. If only the retained degrees of freedom are considered, the resulting improper mixture cannot be distinguished from the analogous proper mixture. But, as d'Espagnat stresses (1995), there is no theoretical reason that prevents from having access to, at least, some of the traced over degrees of freedom: this access would show that the proper mixture and the improper mixture are, in principle, testably different. In the context of decoherence, the difference is also pointed out by Heinz-Dieter Zeh, one of the founding fathers of the decoherence theory: "The conceptually important difference between true and apparent ensembles was clearly pointed out by Bernard d'Espagnat (1976) when he distinguished between proper and improper mixtures [...] this difference can even be observed as recoherence (a relocalization of the superposition, that would be impossible for a proper mixture)." (Zeh 2005: 2).

It is interesting to notice that the discussion about the conceptual difference between proper and improper mixtures makes sense because both are represented by the same kind of mathematical object -a density operator. However, this feature essentially depends on the Hilbert space formalism used to articulate the theory; but the situation is different in other 
formalisms. For instance, proper and improper mixtures are represented by different kinds of density operators in the quaternionic formulation of quantum mechanics (Masillo, Scolarici, and Sozzo 2009, see also Adler 1995); therefore, in this formal framework they can be distinguished not only physically but also mathematically.

On the basis of the above considerations it is quite clear that the reduced state of an open system cannot be strictly considered its quantum state. Nevertheless, this does not imply to deprive the reduced state of any meaning, since it does supply a certain description of the open system. The correspondence between the quantum and the classical domains will offer conceptual elements to elucidate the nature of the reduced state and, with this, to clarify how decoherence should be interpreted.

\section{5.- Analogy and correspondence between classical and quantum domains}

The content of this section will be devoted to show that a general concept of coarse description can be defined such that, both in the classical and in the quantum cases, coarse descriptions result from two related but different operations: coarse-graining and partial trace. The analogy between the classical case and the quantum case will supply the basis to offer, in the next section, a meaningful version of the $\mathrm{CP}$ and, in turn, to understand the physical meaning of decoherence.

\section{1.- Coarse-graining}

In Gibbsian statistical mechanics, the system under study is represented by an ensemble of abstract systems in different microstates that are compatible with the system's macrostate. The statistical state of the system is represented by a density function $\rho(r, t)$-normalized to unity-, defined on a phase space $\Gamma$, where $r \in \Gamma$ represents a possible microstate (position and momentum). In turn, the physical magnitudes depending on the microstates $r$ are represented by observables $O(r)$, such that $O: \Gamma \rightarrow \mathbb{R}$. The density function $\rho(r, t)$ allows computing the phase average (average on all the members of the ensemble) of any observable $O(r)$ as

$$
\langle O(r)\rangle_{\rho(r, t)}=\int_{\Gamma} \rho(r, t) O(r) d r
$$

In this Gibbsian approach, statistical equilibrium is defined as the situation in which the phase averages are independent of time; this situation is represented by the microcanonical ensemble, whose corresponding density distribution is uniform over all the accessible region of the phase space. 
One of the central problems in this theoretical context is how to explain the approach to equilibrium by means of the reversible time-evolution of the density function. In fact, the evolution of $\rho(r, t)$ is ruled by the Liouville equation and, as a consequence, has no limit for $t \rightarrow \infty$ : as proved by the Liouville theorem, the volume of the support of $\rho(r, t)$ in $\Gamma$ is timeinvariant. Therefore, the problem of irreversibility in classical statistical mechanics turns out to be how to account for an irreversible approach to equilibrium in systems ruled by an underlying time-invariant evolution law (see Frigg 2007). The standard answer in the context of the Gibbsian approach relies on coarse graining: the phase space $\Gamma$ is partitioned into cells $C_{i}$ of the same volume $\mu\left(C_{i}\right)$ in $\Gamma$, and a coarse-grained distribution $\rho_{c g}(r)$ is defined such that

$$
\rho_{c g}(r)=\left\{\begin{array}{cc}
\left(1 / \mu\left(C_{1}\right)\right) \int_{C_{1}} \rho(r) d r & \text { if } r \in C_{1} \\
\left(1 / \mu\left(C_{2}\right)\right) \int_{C_{2}} \rho(r) d r & \text { if } r \in C_{2} \\
\vdots &
\end{array}\right.
$$

Since the coarse-grained state $\rho_{c g}$ is not the original statistical state, it is not ruled by the dynamical law of classical statistical mechanics and, as a consequence, its evolution is not constrained by the Liouville theorem: $\rho_{c g}(r, t)$ may approach a definite limit for $t \rightarrow \infty$. This is what effectively happens when the system has a sufficiently high degree of instability: it can be proved that, if the system is mixing, the coarse-grained state approaches a timeindependent equilibrium state, $\rho_{c g}(r, t) \rightarrow \rho_{c g(e q)}(r)$ (see, e.g., Berkovitz, Frigg, and Kronz 2006).

The expectation values of the observables of the system can be computed in the coarsegrained state $\rho_{c g}(r)$ analogously to eq. (6):

$$
\langle O(r)\rangle_{\rho_{c g}(r)}=\int_{\Gamma} \rho_{c g}(r) O(r) d r
$$

It is quite clear that the same result can be obtained if certain "gross observables" are defined as follows:

$$
O_{c g}(r)=\left\{\begin{array}{cc}
\left(1 / \mu\left(C_{1}\right)\right) \int_{C_{1}} O(r) d r & \text { if } r \in C_{1} \\
\left(1 / \mu\left(C_{2}\right)\right) \int_{C_{2}} O(r) d r & \text { if } r \in C_{2} \\
\vdots &
\end{array}\right.
$$

In fact, 


$$
\langle O(r)\rangle_{\rho_{c g}(r)}=\left\langle O_{c g}(r)\right\rangle_{\rho(r)}
$$

In quantum mechanics a similar strategy can be developed. Let us consider a closed system whose Hilbert space $\mathcal{H}$ is spanned by the eigenstates $|r\rangle$ of an observable $R$ of continuous spectrum; so, any state $\hat{\rho}$ and any observable $\hat{O}$ can be expressed, respectively, as

$$
\begin{aligned}
& \hat{\rho}=\iint \rho\left(r, r^{\prime}\right)|r\rangle\left\langle r^{\prime}\right| d r d r^{\prime} \\
& \hat{O}=\iint O\left(r, r^{\prime}\right)|r\rangle\left\langle r^{\prime}\right| d r d r^{\prime}
\end{aligned}
$$

In this case, coarse graining is the result of partitioning the space $\mathcal{H} \otimes \mathcal{H}$ into regions corresponding to $\left(r, r^{\prime}\right) \in C_{i}$. On the basis of this partition, coarse-grained states and coarsegrained observables can be defined as follows. First, certain coarse-grained functions that average the components of states and observables in the regions $C_{i}$ are defined:

$$
\begin{aligned}
& \rho_{c g}\left(r, r^{\prime}\right)=\left\{\iint_{C_{i}} \rho\left(r, r^{\prime}\right)|r\rangle\left\langle r^{\prime}\right| d r d r^{\prime} \quad \text { if }\left(r, r^{\prime}\right) \in C_{i}\right. \\
& O_{c g}\left(r, r^{\prime}\right)=\left\{\iint_{C_{i}} O\left(r, r^{\prime}\right)|r\rangle\left\langle r^{\prime}\right| d r d r^{\prime} \quad \text { if }\left(r, r^{\prime}\right) \in C_{i}\right.
\end{aligned}
$$

Second, the corresponding operators are obtained as

$$
\begin{aligned}
& \hat{\rho}_{c g}=\iint \rho_{c g}\left(r, r^{\prime}\right)|r\rangle\left\langle r^{\prime}\right| d r d r^{\prime} \\
& \hat{O}_{c g}=\iint O_{c g}\left(r, r^{\prime}\right)|r\rangle\left\langle r^{\prime}\right| d r d r^{\prime}
\end{aligned}
$$

This leads to a situation completely analogous to the classical case: the coarse-grained description can be obtained both by averaging the fine-grained observables in the coarsegrained state or by averaging the coarse-grained observables in the fine-grained state (see eq. (10):

$$
\langle\hat{O}\rangle_{\hat{\rho}_{c g}}=\left\langle\hat{O}_{c g}\right\rangle_{\hat{\rho}}
$$

This quantum coarse-graining procedure was the strategy followed in certain early attempts to explain the emergence of classicality during a period that can be considered as the pre-history of the decoherence program. In the 1950s and the early 1960s, the issue was treated in the context of the study of irreversibility in closed systems (van Kampen 1954, van Hove 1957, 1959, Daneri, Loinger, and Prosperi 1962). From this perspective, the states that are indistinguishable from the viewpoint of certain "gross" observables are described by the same coarse-grained state, whose evolution can be proved to reach equilibrium in a certain 
relaxation time. The main problem of this period was that the relaxation times so obtained turned out to be too long to account for the experimental emergence of classical behavior (see Omnès 2005).

Summing up, although coarse graining is a traditional procedure to explain irreversibility in classical statistical mechanics, it can be appealed to when the issue is quantum irreversibility; see Table 1 for the analogy between the two cases (Fortin and Lombardi 2018).

\begin{tabular}{|c|c|}
\hline CSM & QM \\
\hline$r \in \Gamma ; \rho(r) ; O(r)$ & $R$ with $|r\rangle ; \hat{\rho} ; \hat{O}$ \\
\hline Coarse graining in variable $r$ & Coarse graining in variable $r$ \\
$\rho_{c g}(r)=\left\{\begin{array}{cc|}\frac{1}{\mu\left(C_{i}\right)} \int_{C_{i}} \rho(r) d r \text { if } r \in C_{i} & \rho_{c g}\left(r, r^{\prime}\right)=\left\{\iint_{C_{i}} \rho\left(r, r^{\prime}\right) d r d r^{\prime} \text { if }\left(r, r^{\prime}\right) \in C_{i}\right. \\
O_{c g}(r)=\left\{\frac{1}{\mu\left(C_{i}\right)} \int_{C_{i}} O(r) d r \text { if } r \in C_{i}\right. & \hat{\rho}_{c g}=\iint \rho_{c g}\left(r, r^{\prime}\right)|r\rangle\left\langle r^{\prime}\right| d r d r^{\prime} \\
\hat{O}_{c g}=\iiint_{C_{i}} O\left(r, r^{\prime}\right) d r d r^{\prime} \text { if }\left(r, r^{\prime}\right) \in C_{i} \\
O_{c g}\left(r, r^{\prime}\right)|r\rangle\left\langle r^{\prime}\right| d r d r^{\prime}\end{array}\right.$ \\
\hline$\langle O\rangle_{\rho_{c g}}=\left\langle O_{c g}\right\rangle_{\rho}$ & $\langle\hat{O}\rangle_{\hat{\rho}_{c g}}=\left\langle\hat{O}_{c g}\right\rangle \hat{\rho}$ \\
\hline
\end{tabular}

Table 1: Coarse graining in classical statistical mechanics and in quantum mechanics.

\section{2.- Partial trace}

Although the reduced state of an open system can be computed by tracing over the degrees of freedom of its environment, the very definition of the concept of reduced state does not appeal to partial trace. The reduced state $\hat{\rho}_{r}^{S}$ of the open system $S$ is defined as the density operator by means of which the expectation values of all the observables of $S$ can be computed. Precisely, if $\mathcal{H}_{S}$ and $\mathcal{H}_{E}$ are the Hilbert spaces of the open system $S$ and its environment $E$ respectively, $\mathcal{H}=\mathcal{H}_{S} \otimes \mathcal{H}_{E}$ is the Hilbert space of the closed system $S E, \hat{O}_{S} \in \mathcal{H}_{S} \otimes \mathcal{H}_{S}$ is an observable of $S, \hat{I}_{E}$ is the identity in $\mathcal{H}_{E} \otimes \mathcal{H}_{E}$, and $\hat{\rho} \in \mathcal{H} \otimes \mathcal{H}$ is the state of $S E$, then the reduced state of $S$ is defined as the density operator $\hat{\rho}_{r}^{S}$ such that

$$
\left\langle\hat{O}_{S}\right\rangle_{\hat{\rho}_{r}^{S}}=\left\langle\hat{O}_{S}^{S E}\right\rangle_{\hat{\rho}}
$$


where $\hat{O}_{S}^{S E}=\hat{O}_{S} \otimes \hat{I}_{E}$. On the basis of this definition, the reduced state can be computed by tracing over the degrees of freedom of $E$, as $\hat{\rho}_{r}^{S}=\operatorname{Tr}_{E} \hat{\rho}$.

In the quantum domain, the operation of partial-trace was used not only in the account of the phenomenon of decoherence. It was already implicit in what Erwin Schrödinger (1926) called "density of electricity" or "electric charge density", a quantity defined in the space of three dimensions, which he considered the quantum magnitude with direct physical meaning (see Przibram 1967).

In classical statistical mechanics an analogous strategy can be developed. Let us consider a closed system $S E$ of $N$ particles described by a phase space $\Gamma$ of $6 N$ dimensions. The states and observables of the system are functions of the $3 N$ positions $q_{i}$ and the $3 N$ momenta $p_{i}$ of the $N$ particles:

$$
\begin{aligned}
& \rho(r)=\rho\left(q_{1}, p_{1}, \ldots, q_{3 N}, p_{3 N}\right) \\
& O(r)=O\left(q_{1}, p_{1}, \ldots, q_{3 N}, p_{3 N}\right)
\end{aligned}
$$

If the purpose is to describe an open subsystem $S$ of $s$ particles, the interest is centered on the observables $O_{S}\left(q_{1}, p_{1}, \ldots, q_{3 s}, p_{3 s}\right)$. In order to compute the expectation values of these observables, a reduced state $\rho_{r}^{S}(r)$ can be computed by means of a partial trace:

$$
\rho_{r}^{S}\left(q_{1}, p_{1}, \ldots, q_{3 s}, p_{3 s}\right)=\int \ldots \int \rho\left(q_{1}, p_{1}, \ldots, q_{3 N}, p_{3 N}\right) d q_{3 s+1} d p_{3 s+1} \ldots d q_{3 N} d p_{3 N}
$$

The reduced state $\rho_{r}^{S}\left(q_{1}, p_{1}, \ldots, q_{3 s}, p_{3 s}\right)$ of the subsystem $S$ of $s$ particles disregards the degrees of freedom of the environment $E$ composed by the remaining $N-s$ particles. In turn, the observable of the open system $S$ can be expressed from the viewpoint of the closed system of $N$ particles as a function on the phase space $\Gamma$ :

$$
O_{S}\left(q_{1}, p_{1}, \ldots, q_{3 s}, p_{3 s}\right)=O_{S}^{S E}\left(q_{1}, p_{1}, \ldots, q_{3 N}, p_{3 N}\right)
$$

The reduced state $\rho_{r}^{S}\left(q_{1}, p_{1}, \ldots, q_{3 s}, p_{3 s}\right)$ can also be expressed from the viewpoint of the closed system as a function on $\Gamma$ :

$$
\rho_{r}^{S}\left(q_{1}, p_{1}, \ldots, q_{3 s}, p_{3 s}\right)=\rho_{S}^{S E}\left(q_{1}, p_{1}, \ldots, q_{3 N}, p_{3 N}\right)
$$

Therefore, the expectation values of the observables of $S$ can be computed alternatively from the perspective of the closed system $S E$ of $N$ particles or from the perspective of the open subsystem $S$ of $s$ particles (see the analogy with the quantum case given by eq. (18)):

$$
\left\langle O_{S}\right\rangle_{\rho_{r}^{s}}=\left\langle O_{S}^{S E}\right\rangle_{\rho}
$$


In this classical statistical context, reduced densities are used to compute the expectation values of the observables (phase functions) that depend on the dynamical variables of a subset of the particles of the system, such as, for instance, the kinetic energy of a single particle, or the potential energy of two particles. The evolution of the reduced densities is described by the set of equations known as BBGKY hierarchy due to the name of its authors: Nicolái Bogoliubov (1946), Max Born and Herbert Green (1946), John Kirkwood (1946), and Jacques Yvon (1935).

Summing up, although partial trace is a traditional procedure to account for the behavior of open systems in quantum mechanics, it is also used in classical statistical mechanics when the interest is focused on certain particular magnitudes; see Table 2 for the analogy between the two cases (Fortin and Lombardi 2018).

\begin{tabular}{|c|c|}
\hline CSM & QM \\
\hline System $S E: \Gamma(N$ particles $) ; \rho ; O$ & System $S E: \mathcal{H} \otimes \mathcal{H} ; \hat{\rho} ; \hat{O}$ \\
\hline Subsystem $S(s$ particles $)$ & Subsystem $S$ (open system) \\
$O_{S}\left(q_{1}, p_{1}, \ldots, q_{3 s}, p_{3 s}\right)$ & $\hat{O}_{S} \in \mathcal{H}_{S} \otimes \mathcal{H}_{S}$ \\
$O_{S}^{S E}\left(q_{1}, p_{1}, \ldots, q_{3 N}, p_{3 N}\right)$ & $\hat{O}_{S}^{S E}=\hat{O}_{S} \otimes \hat{I}_{E}$ \\
$\rho_{r}^{S}=\int \ldots . . \rho d q_{3 s+1} d p_{3 s+1} \ldots d q_{3 N} d p_{3 N}$ & $\hat{\rho}_{r}^{S}=T r_{E} \hat{\rho}$ \\
\hline$\left\langle O_{S}\right\rangle_{\rho_{r}^{S}}=\left\langle O_{S}^{S E}\right\rangle_{\rho}$ & $\left\langle\hat{O}_{S}\right\rangle_{\hat{\rho}_{r}^{S}}=\left\langle\hat{O}_{S}^{S E}\right\rangle_{\hat{\rho}}$ \\
\hline
\end{tabular}

Table 2: Partial trace in classical statistical mechanics and in quantum mechanics.

\section{3.- Coarse descriptions}

As explained in Subsection 5.1, coarse-grained descriptions result from a partition of the space of states into discrete and disjoint cells $C_{i}$ : this operation defines the coarse-grained distribution. Since applying the same coarse-graining operation to the coarse-grained distribution does not modify the result, the relationship between the coarse-grained and the fine-grained descriptions can be described in terms of projection:

$$
\begin{array}{ll}
\text { classical: } & \rho_{c g}=\Pi^{\mathrm{cg}} \rho=\Pi^{\mathrm{cg}} \Pi^{\mathrm{cg}} \rho \\
\text { quantum: } & \hat{\rho}_{c g}=\hat{\Pi}^{\mathrm{cg}} \hat{\rho}=\hat{\Pi}^{\mathrm{cg}} \hat{\Pi}^{\mathrm{cg}} \hat{\rho}
\end{array}
$$


where the projectors $\Pi^{\mathrm{cg}}$ and $\hat{\Pi}^{\mathrm{cg}}$ perform the operations described by eq. (7) and eq. (15), respectively (strictly speaking, in the Hilbert-space formalism of Koopman (1931) in the classical case).

On the other hand, as explained above, the reduced state of an open system $S$ in interaction with its environment $E$ is defined as the state by means of which the expectation values of all the observables of $S$ can be computed. But, in this case, the state of the closed composite system $S E$ and the reduced state of $S$ belong to different mathematical spaces. Therefore, for dimensional reasons the reduced state of $S$ cannot be directly expressed as the result of a projection onto the state of $S E$. Nevertheless, a coarse description from the viewpoint of the closed composite system can be defined in the following way (the explanation corresponds to the quantum case, but it is completely analogous for the classical case in the formalism of Koopman). The expectation value of $\hat{O}_{S}$ in the reduced state $\hat{\rho}_{r}^{S}$, originally computed as in eq. (18), can also be expressed as the expectation value of the observables $\hat{O}_{S}^{S E}=\hat{O}_{S} \otimes \hat{I}_{E}$ of the closed system $S E$ in a state $\hat{\rho}_{r}^{S E} \in \mathcal{H} \otimes \mathcal{H}$ :

$$
\left\langle\hat{O}_{S}\right\rangle_{\hat{\rho}_{r}^{S}}=\left\langle\hat{O}_{S}^{S E}\right\rangle_{\hat{\rho}_{r}^{S E}}=\left\langle\hat{O}_{S} \otimes \hat{I}_{E}\right\rangle_{\hat{\rho}_{r}^{S E}}
$$

The density operator $\hat{\rho}_{r}^{S E}$ can be obtained as

$$
\hat{\rho}_{r}^{S E}=\hat{\Pi}^{\mathrm{pt}} \hat{\rho}=\hat{\Pi}^{\mathrm{pt}} \hat{\Pi}^{\mathrm{pt}} \hat{\rho}
$$

where the projector $\hat{\Pi}^{\mathrm{pt}}$ performs the following operation:

$$
\hat{\Pi}^{\mathrm{pt}} \hat{\rho}=\left(\operatorname{Tr}_{E} \hat{\rho}\right) \otimes \tilde{\delta}_{E}=\hat{\rho}_{r}^{S} \otimes \tilde{\delta}^{E}
$$

and $\tilde{\delta}^{E} \in \mathcal{H}_{E} \otimes \mathcal{H}_{E}$ is a normalized identity operator with coefficients $\tilde{\delta}_{\alpha \beta}^{E}=\delta_{\alpha \beta} / \sum_{\gamma} \delta_{\gamma \gamma}$ (see Fortin and Lombardi 2014). In turn, if we trace off the degrees of freedom of the environment $E$ on $\hat{\rho}_{r}^{S E}$, we recover the reduced state of the open system $S$ :

$$
\operatorname{Tr}_{E} \hat{\rho}_{r}^{S E}=\operatorname{Tr}_{E}\left(\hat{\rho}_{r}^{S} \otimes \tilde{\delta}^{E}\right)=\hat{\rho}_{r}^{S}
$$

This means that the $\hat{\rho}_{r}^{S E}$, which "erases" the components of the quantum state $\hat{\rho}$ corresponding to the environment $E$, supplies the same information about the open system $S$ as the reduced state $\hat{\rho}_{r}^{S}$, but now from the viewpoint of the closed system $S E$. Therefore, the relationship between the traced and the non-traced description can also be described in terms of projection:

$$
\begin{array}{ll}
\text { classical: } & \rho_{r}^{S E}=\Pi^{\mathrm{pt}} \rho=\Pi^{\mathrm{pt}} \Pi^{\mathrm{pt}} \rho \\
\text { quantum: } & \hat{\rho}_{r}^{S E}=\hat{\Pi}^{\mathrm{pt}} \hat{\rho}=\hat{\Pi}^{\mathrm{pt}} \hat{\Pi}^{\mathrm{pt}} \hat{\rho}
\end{array}
$$


Summing up, both in the case of coarse graining and in the case of partial trace, a state that supplies a coarse description of the closed system can be defined, in such a way that it can be obtained as a projection onto the "fine description". The difference between the two cases lies in what is disregarded in each one of them. As clearly explained by Michael Mackey (1989), coarse graining implies the loss of some information about the exact values of all the dynamical variables, whereas partial trace implies the loss of all the information about the exact value of some dynamical variables.

Table 1 and Table 2, as well as the two pairs of equations (25)-(26) and (31)-(32) show relevant analogies between the classical and the quantum domains concerning expectation values. These analogies can be regarded as a case of what Stephan Hartmann (2008) calls 'structure correspondence', in his systematization of the different types of correspondence relations. In the next section we will show how this structure correspondence can be conceived as a particular case of a generalized version of the correspondence principle.

\section{6.- Correspondence and the physical meaning of decoherence}

\section{1.- Generalizing the Correspondence Principle}

Although the original CP was designed to establish a link between frequencies and motions, the idea was broadened already in Bohr's times. In fact, in his doctoral dissertation under Sommerfeld's supervision, Pauli (1922) introduced what he called a "mechanical" CP: since classical mechanics applied to inelastic collision processes should also break down because of energy quantization, a CP for this case, in analogy with Bohr's electrodynamical principle, was necessary.

Of course, in Pauli's extrapolation to the mechanical domain, the CP has not exactly the original meaning devised by Bohr. Nevertheless, the "mechanical CP" still retains the idea of a certain correspondence between quantum and classical. This extrapolation suggests the possibility of formulating a generalized version of the $C P$, concerned with the correspondence between analogous quantum and classical concepts and regularities. In the light of this proposal, the structural correspondence between the classical and the quantum domains concerning expectation values, as described in Section 5, turns out to be a particular case of that generalized version of Bohr's CP. On this basis, two remarks are in order.

First, it is worth emphasizing again that the structural correspondence appears at the level of the expectation values. In the early days of the environment-induced program, and following the works of other authors (e.g., Ballentine, Yang, and Zibin 1994), some 
decoherence theorists conceived the $\mathrm{CP}$ as the demand for the agreement between quantum and classical expectation values (see, e.g., Habib, Shizume, and Zurek 1998), and claimed that decoherence is the physical process that leads to such an agreement. But this expectationvalue perspective got lost in later works, in which the phenomenon of decoherence came to be seen as the result of the dynamical evolution of the open system's reduced state. In the light of the structure correspondence exhibited in the previous section, here we will come back to the perspective based on expectation values in order to understand decoherence.

Second, and related with the previous remark: from the perspective opened by structure correspondence, this particular case of the generalized CP becomes an expectation-value requirement, that is, the demand for the agreement between classical and quantum expectation values. This is the view adopted by Belot and Earman when, in their article about the CP in the context of the problem of quantum chaos, they require "that the quantum expectation values mimic classical expectation values computed from the appropriate $\rho . "(1997: 169)$. It is precisely in this point that quantum decoherence plays a substantial role: the agreement required by the $\mathrm{CP}$ is reached only when decoherence occurs. In other words, decoherence is the phenomenon that allows the $\mathrm{CP}$, in its expectation-value version, to be fulfilled. And, in turn, as will be argued below, this version of the $\mathrm{CP}$ offers the conceptual elements to understand the physical meaning of decoherence.

\section{2.- Using the Correspondence Principle as a conceptual tool}

At this point we have all the elements to follow Batterman's strategy: appealing to the $\mathrm{CP}$ as a guiding principle to face conceptual problems related with the relation between classical and quantum. In particular, the $\mathrm{CP}$ as a conceptual tool will help us to elucidate the nature of the reduced state and, with it, the physical meaning of the phenomenon of decoherence.

In the classical case, the diverse ways of conceiving the coarse-grained state express the different interpretations of irreversibility, in the context of a debate that is still as alive as in the birth of statistical mechanics (see, e.g. Frigg 2007). Nevertheless, despite the debates about interpretation, nobody ignores the difference between the statistical state, which evolves unitarily according to the dynamical postulate of the theory, and the coarse-grained state, which may approach a final stable state. Even those who take a heterodox position by claiming the priority of the coarse-grained state regarding objectivity are clear on that such a move requires a reformulation of the fundamental dynamical law of statistical mechanics (see, e.g., Misra, Prigogine, and Courbage 1979, Nicolis and Prigogine 1989). In other words, independently of the particular disagreements about irreversibility, nobody attempts to endow 
the statistical state and the coarse-grained state with the same interpretation. On the basis of the generalized version of the $\mathrm{CP}$, given that in the quantum case the reduced state is also a coarse description regarding the fine description, there is no reason to conflate the reduced state of the open system with its quantum state.

As a consequence, both in the classical and in the quantum cases, the system's state, which evolves according to the dynamical postulate of the respective theory, must not be confused with a coarse-grained state or a reduced state, which offers a coarse description of the system. Once it is accepted that $\rho_{c g}$ and $\rho_{r}^{S}$ are not classical-statistical states, it must be admitted that $\hat{\rho}_{c g}$ and $\hat{\rho}_{r}^{S}$ are not quantum states either. This is particularly relevant for the interpretation of quantum mechanics, since endowing reduced states with the same interpretation as quantum states yields interpretive difficulties, in particular, is an obstacle for no-collapse interpretations to explain consecutive measurements (Ardenghi, Lombardi, and Narvaja 2012). When the coarse nature of $\rho_{r}^{S}$ is accepted, the decoherence of the reduced state is a phenomenon that proceeds at a coarse level of description, analogously to the case of classical irreversibility. In fact, as Roland Omnès $(2001,2002)$ stresses, decoherence is a case of quantum irreversibility.

In turn, describing decoherence as a coarse-level phenomenon amounts to interpreting it as a process that happens to expectation values: the description in terms of reduced state is only a shortcut to explain an expectation-value process. More precisely, when considered from a fundamental viewpoint, decoherence involves the convergence of the expectation value of any observable of the open system to a value that can be computed as if the system were closed and in a state represented by a diagonal density operator.

$$
\left\langle\hat{O}_{S}^{S E}\right\rangle_{\hat{\rho}(t)}=\left\langle\hat{O}_{S}\right\rangle_{\hat{\rho}_{r}^{S}(t)} \longrightarrow\left\langle\hat{O}_{S}\right\rangle_{\hat{\rho}_{r}^{S}}
$$

As Maximilian Schlosshauer claims in his well-known book about decoherence, strictly speaking a reduced density operator is only "a calculational tool" for computing expectation values (Schlosshauer 2007: 48). For this reason, the author warns us "against a misinterpretation of reduced density matrices as describing a proper mixture of states" (2007: 69).

This view of decoherence based on the expectation-value perspective contrasts with Zurek's reading of the phenomenon. Zurek embraces a dynamical interpretation, according to which decoherence is a process that transforms quantum states, turning pure states into mixtures. From the expectation-value perspective, decoherence is a phenomenon that involves 
reduced states, which are not quantum quantum states but coarse states. An it is precisely the parallel with the classical case, brought to light by the quantum-classical structural correspondence, that supports this view that clearly distinguishes between quantum state and reduced state.

Summing up, the expectation-value version of the generalized CP backs up an interpretation of the phenomenon of decoherence different from the dynamical approach implicit in most presentations: since the reduced state is not the quantum state of the open system, but a coarse description of the closed composite system, decoherence should not be conceived as a phenomenon resulting from the interaction between an open system and its environment (see Castagnino, Fortin, and Lombardi 2010a). By contrast, decoherence is a coarse process that emerges, under appropriate conditions, from taking into account only the evolution of some degrees of freedom of the whole closed system and disregarding the remaining degrees of freedom conceived as the environment (see Fortin and Lombardi 2016).

\section{7.- Conclusions and perspectives}

In this article, we began by recalling the first versions of the $\mathrm{CP}$ in old quantum theory, showing how the original claim about a link between frequencies and motions in the atom gradually turned out to be conceived by physicists as a requirement of the classical limit of quantum mechanics. Once the $\mathrm{CP}$ and the demand for the classical limit of quantum mechanics are distinguished, a principle proposed in the context of the old quantum theory seems to have no other relevance to present-day physics than a historical interest. In contrast with this view, we claimed that the CP can still be useful to elucidate the relationship between the quantum and the classical domains. In order to argue for this position, we recalled Robert Batterman's proposal of appealing to the $\mathrm{CP}$, in its original formulation, as a conceptual resource to face the hard problem of defining quantum chaos.

On the one hand, inspired by Batterman's strategy, we undertook the task of elucidating the concept of decoherence in the light of the CP. Since the core of the meaning of decoherence lies in the concept of reduced state, we developed a detailed structural correspondence between quantum mechanics and classical statistical mechanics in order to elucidate that concept. Such a correspondence made clear that, both in the classical and in the quantum cases, the coarse-grained state and the partial-traced state supply coarse descriptions, in such a way that they can be obtained as projections of the corresponding "fine description". In turn, this is a manifestation of the fact that the quantum-classical structural correspondence holds at the level of expectation values, 
On the other hand, Pauli's mechanical extrapolation of the CP led us to formulate a generalized $\mathrm{CP}$, concerned with the correspondence between analogous quantum and classical concepts and regularities. Therefore, the $\mathrm{CP}$, as the demand for the agreement between classical and quantum expectation values, turns out to be a particular case of that generalized version of the CP. In this context, decoherence is the phenomenon that allows the $\mathrm{CP}$, under this expectation-value form, to be fulfilled. As a consequence, decoherence must be understood as a process that occurs at the level of the expectation values; the reduced state must be viewed as a calculation tool for computing the expectation values of interest. This means that decoherence is a coarse phenomenon resulting from taking into account only the evolution of some degrees of freedom of the whole closed system and disregarding the remaining degrees of freedom conceived as the environment. In other words, it is a phenomenon relative to the observables selected to be studied. This way of understanding decoherence, devised under the guide of the $\mathrm{CP}$, is completely different than the dynamical reading based on assuming the transformation of pure states in mixtures, typical in the writings of the main theorists of environment-induced approach to decoherence.

This understanding of decoherence offers a simple way out for the difficulties that challenge the orthodox environment-induced approach, which are byproducts of interpreting reduced states as quantum states (see Castagnino, Laura and Lombardi 2007):

- The "open-system" problem: If only open systems may decohere, the issue of the emergence of classicality in closed systems, in particular, in the universe as a whole, cannot even be posed. In Zurek's words: "the Universe as a whole is still a single entity with no 'outside' environment, and, therefore, any resolution involving its division into systems is unacceptable." (1994: 181). This difficulty has led to the development of non-dissipative approaches to decoherence (see, e.g., Bonifacio et al. 2000, Ford and O'Connell 2001, Frasca 2003).

This "open-system" problem vanishes from the new perspective. Now decoherence is no longer conceived as the result of the interaction of an open system with its environment. When understood as a coarse evolution, as in the case of classical irreversibility, decoherence may happen in closed systems regarding certain relevant observables (this closed-system view leads to a generalized view of decoherence, see Fortin, Lombardi, and Castagnino 2014, Fortin and Lombardi 2016).

- The "defining-system" problem: The orthodox approach offers no general criterion to place the "cut" between system and environment: given the possibility of "internal" environments, the partition must be decided case by case, and usually depends on the 
previous assumption of the observables that will behave classically. In Zurek's words: "In particular, one issue which has been often taken for granted is looming big as a foundation of the whole decoherence program. It is the question of what are the 'systems' which play such a crucial role in all the discussions of the emergent classicality. This issue was raised earlier, but the progress to date has been slow at best." (1998: 22).

The "defining-system" problem is simply dissolved by the expectation-value view. In fact, any coarse description depends on how the relevant information is selected. As a consequence, decoherence is relative to the relevant observables selected to be studied (Castagnino, Fortin, and Lombardi 2010b, Dugić and Jeknić-Dugić 2012, Lychkovskiy 2013). In turn, since there is no privileged or essential decomposition of the closed system (Harshmann and Wickramasekara 2007a, 2007b, Harshman 2019), there is no need for an unequivocal criterion to identify the systems involved in decoherence (for a detailed discussion, see Lombardi, Fortin, and Castagnino 2012).

The interpretation of decoherence as a relative phenomenon can be generalized leading to a closed-system view of quantum mechanics, also inspired in the classical-quantum correspondence. In the case of classical statistical mechanics, the dynamical postulate -the Liouville equation- is applied to closed systems, which can be also approached from different coarse descriptions. Analogously, the only legitimate quantum systems are the unitary evolving closed systems, which can be coarsely described in many different ways. This view finds a significant affinity with the so called "quantum structure studies", dealing with the different ways in which a quantum system can be decomposed into subsystems (quantum structures) (see, e.g., Zanardi 2001, Jeknić-Dugić, Arsenijević, and Dugić 2013). From this closed-system perspective, entanglement is also relative to the partition of the closed system into parts (Barnum et al. 2003, 2004, Viola and Barnum 2010), and this relativity dissolves what John Earman (2014) calls "puzzles about quantum entanglement", derived from the lack of a univocal criterion to decompose a closed quantum system.

These recent works show that the $\mathrm{CP}$, with no need of being interpreted in terms of a quantum-to-classical limit but conceived as a guiding principle regarding the relations between the classical and the quantum domains, may continue to offer relevant contributions to the understanding of quantum mechanics.

\section{References}

Adler, S. L. (1995). Quaternionic Quantum Mechanics and Quantum Fields. New York: Oxford University Press. 
Ballentine, L., Yang, Y., and Zibin, J. (1994). "Inadequacy of Ehrenfest's theorem to characterize the classical regime." Physical Review A, 50: 2854-2859.

Barnum, H., Knill, E., Ortiz, G., Somma, R. and Viola, L. (2003). "Generalizations of entanglement based on coherent states and convex sets." Physical Review A, 68: 032308 .

Barnum, H., Knill, E., Ortiz, G., Somma, R. and Viola, L. (2004). “A subsystem-independent generalization of entanglement." Physical Review Letters, 92: 107902.

Batterman, R. (1991). “Chaos, quantization, and the correspondence principle.” Synthese, 89: 189-227.

Batterman, R. (1993). “Defining chaos.” Philosophy of Science, 60: 43-66.

Belot, G. and Earman, J. (1997). "Chaos out of order: Quantum mechanics, the correspondence principle and chaos." Studies in History and Philosophy of Modern Physics, 28: 147-182.

Berkovitz, J., Frigg, R., and Kronz, F. (2006). "The ergodic hierarchy, randomness and Hamiltonian chaos." Studies in History and Philosophy of Modern Physics, 37: 661691.

Berry, M. V. (1983). "Semi-classical mechanics of regular and irregular motion." Pp. 171.271 in G. Ioos, R. G. H. Helleman, and R. Stora (eds.), Chaotic Behaviour of Deterministic Systems (Les Houches, Session 36). Amsterdam: North-Holland.

Berry, M. V. (1989). “Quantum chaology, not quantum chaos.” Physica Scripta, 40: 335-336.

Bogoliubov, N. N. (1946). "Kinetic equations." Journal of Experimental and Theoretical Physics, 16: 691-702.

Bohm, D. (1951). Quantum Theory. New York: Prentice Hall.

Bohr, N. (1913). "On the constitution of atoms and molecules." Philosophical Magazine, 26: $1-25,476-502,857-75$.

Bohr, N. (1918). "The quantum theory of line-spectra." Det Kongelige Danske Videnskabernes Selskab, Matematisk-Fysiske Meddelelser, 4: 1-36. Page numbers taken from Bohr (1976), pp. 67-102.

Bohr, N. (1920). "On the series spectra of the elements." Lecture before the German Physical Society in Berlin (27 April 1920). Page numbers taken from the translation by A. D. Udden in Bohr (1976), pp. 241-282.

Bohr, N. (1924). "On the application of the quantum theory to atomic structure." Pp. 1-42 in Proceedings of the Cambridge Philosophical Society (supplement). Cambridge: Cambridge University Press. First published in 2023, Zeitschrift für Physik, 13: 117165. Reprinted in Bohr (1976), pp. 457-499.

Bohr, N. (1976). Niels Bohr Collected Works, Vol. 3: The Correspondence Principle (19181923), J. R. Nielsen (ed.). Amsterdam: North-Holland Publishing.

Bokulich, A. (2014). “Bohr's correspondence principle.” In E. N. Zalta (ed.), The Stanford Encyclopedia of Philosophy (Spring 2014 Edition). https://plato.stanford.edu/archives/spr2014/entries/bohr-correspondence/

Bonifacio, R., Olivares, S., Tombesi, P., and Vitali, D. (2000). "Model-independent approach to nondissipative decoherence." Physical Review A, 61: 053802. 
Born, M. (1933). Moderne Physik. Berlin: Julius Springer. Page numbers taken from the English version of 1957, Atomic Physics, $6^{\text {th }}$ Edition, J. Dougall and R. Blin-Stoyle (trans.). New York: Hafner Publishing Co.

Born, M. and Green, H. S. (1946). "A general kinetic theory of liquids I. The molecular distribution functions." Proceedings of the Royal Society A, 188: 10-18.

Castagnino, M., Fortin, S., and Lombardi, O. (2010a). "Is the decoherence of a system the result of its interaction with the environment?" Modern Physics Letters A, 25: 14311439.

Castagnino, M., Fortin, S., and Lombardi, O. (2010b). "Suppression of decoherence in a generalization of the spin-bath model." Journal of Physics A: Mathematical and Theoretical, 43: 065304.

Castagnino, M., Laura, R., and Lombardi, O. (2007). "A general conceptual framework for decoherence in closed and open systems." Philosophy of Science, 74: 968-980.

Daneri, A., Loinger, A., and Prosperi, G. M. (1962). "Quantum theory of measurement and ergodicity conditions." Nuclear Physics, 33: 297-319.

d'Espagnat, B. (1976). Conceptual Foundations of Quantum Mechanics. Reading MA: Addison-Wesley.

d'Espagnat, B. (1995). Veiled Reality. An Analysis of Present-Day Quantum Mechanical Concepts. Reading MA: Addison-Wesley.

Dirac, P. A. M. (1925). "The fundamental equations of quantum mechanics." Proceedings of the Royal Society of London, 109: 642-653.

Dugić, M. and J. Jeknić-Dugić, J. (2012). "Parallel decoherence in composite quantum systems." Pramana Journal of Physics, 79: 199-2019.

Earman, J. (2014). "Some puzzles and unresolved issues about quantum entanglement." Erkenntnis, 80: 303-337.

Ford, J., Mantica, G., and Ristow, G. H. (1991). "The Arnol'd cat: Failure of the correspondence principle." Physica D, 50: 493-520.

Ford, J. and Mantica, G. (1992). "Does quantum mechanics obey the correspondence principle? Is it complete?" American Journal of Physics, 60: 1086-1098.

Ford, G. and O'Connell, R. (2001). "Decoherence without dissipation." Physics Letters A, 286: 87-90.

Fortin, S. and Lombardi, O. (2014). "Partial traces in decoherence and in interpretation: What do reduced states refer to?" Foundations of Physics, 44: 426-446.

Fortin, S. and Lombardi, O. (2016). "A top-down view of the classical limit of quantum mechanics.” Pp. 435-468 in R. Kastner, J. Jeknić-Dugić, and G. Jaroszkiewicz (eds.), Quantum Structural Studies: Classical Emergence from the Quantum Level. Singapore: World Scientific.

Fortin, S. and Lombardi, O. (2018). "Understanding decoherence as an irreversible process." International Journal of Quantum Foundations, 4: 247-267.

Fortin, S., Lombardi, O., and Castagnino, M. (2014). "Decoherence: A closed-system approach.” Brazilian Journal of Physics, 44: 138-153. 
Frasca. M. (2003). "General theorems on decoherence in the thermodynamic limit." Physics Letters A, 308: 135-139.

Frigg, R. (2007). "A field guide to recent work on the foundations of thermodynamics and statistical mechanics." Pp. 99-196 in D. Rickles (ed.), The Ashgate Companion to the New Philosophy of Physics. London: Ashgate.

Habib, S., Shizume, K, and Zurek, W. (1998). "Decoherence, chaos, and the correspondence principle." Physical Review Letters, 80: 4361-4365.

Harshman, N. (2019). "Symmetry, structure, and emergent subsystems." Forthcoming in O. Lombardi, S. Fortin, C. López and F. Holik (eds.), Quantum Worlds. Perspectives on the ontology of quantum mechanics. Cambridge: Cambridge University Press.

Harshman, N. and Wickramasekara, S. (2007a). "Galilean and dynamical invariance of entanglement in particle scattering." Physical Review Letters, 98: 080406.

Harshman, N. and Wickramasekara, S. (2007a). "Tensor product structures, entanglement, and particle scattering." Open Systems and Information Dynamics, 14: 341-351.

Hartmann, S. (2008). "Modeling high-temperature superconductivity: Correspondence at bay?" pp. 109-129 in L. Soler, H. Sankey, and P. Hoyningen-Huene (eds.), Rethinking Scientific Change and Theory Comparison: Stabilities, Ruptures, Incommensurabilities. Dordrecht: Springer.

Heilbron, J. (1983). "The origins of the exclusion principle." Historical Studies in the Physical Sciences, 13: 261-310.

Jeknić-Dugić, J., Arsenijević, M. and Dugić, M. (2013). Quantum Structures: A View of the Quantum World. Saarbrücken: Lambert Academic Publishing.

Kirkwood, J. (1946). "The statistical mechanical theory of transport processes I. General theory." The Journal of Chemical Physics, 14: 180-201.

Koopman, B. O. (1931). "Hamiltonian systems and transformations in Hilbert space." Proceedings of the National Academy of Sciences, 18: 315-318.

Kramers, H. (1919). "Intensities of spectral lines. On the application of the quantum theory to the problem of the relative intensities of the components of the fine structure and of the Stark effect of the lines of the hydrogen spectrum." Det Kongelige Danske Videnskabernes Selskabs Skrifter. Naturvidenskabelig og Mathematisk Afdeling, Copenhagen.

Kramers, H. (1923). "Das Korrespondenzprinzip und der Schalenbau des Atoms." Die Naturwissenschaften, 27: 550-559.

Kronz, F. (1998). "Nonseparability and quantum chaos.” Philosophy of Science, 65: 50-75.

Lychkovskiy, O. (2013). "Dependence of decoherence-assisted classicality on the way a system is partitioned into subsystems." Physical Review A, 87: 022112.

Lombardi, O., Fortin, S., and Castagnino, M. (2012). "The problem of identifying the system and the environment in the phenomenon of decoherence." Pp. 161-174 in H. de Regt, S. Okasha, and S. Hartmann (eds.), EPSA Philosophy of Science: Amsterdam 2009. Dordrecht: Springer.

Mackey, M. C. (1989). "The dynamic origin of increasing entropy." Review of Modern Physics, 61: 981-1015. 
Masillo, F., Scolarici, G., and Sozzo, S. (2009). "Proper versus improper mixtures: towards a quaternionic quantum mechanics." Theoretical and Mathematical Physics, 160: 10061013.

Misra. B., Prigogine, I., and Courbage, M. (1979). "From deterministic dynamics to probabilistic descriptions." Physica A, 98: 1-26.

Nicolis, G. and Prigogine, I. (1989). Exploring Complexity. An Introduction. New York: Freeman \& Company.

Omnès, R. (2001). "Decoherence: an irreversible process." Los Alamos National Laboratory, arXiv:quant-ph/0106006.

Omnès, R. (2002). "Decoherence, irreversibility and the selection by decoherence of quantum states with definite probabilities." Physical Review A, 65: 052119.

Omnès, R. (2005). "Results and problems in decoherence theory." Brazilian Journal of Physics, 35: 207-210.

Pauli, W. (1922). "Über das Modell des Wasserstoffmolekuelions." Annalen der Physik, 68: 177-240. "Improved and expanded" version of Pauli's doctoral dissertation under Sommerfeld.

Paz, J. P. and Zurek, W. H. (2002). "Environment-induced decoherence and the transition from quantum to classical." Pp. 77-148 in D. Heiss (ed.), Fundamentals of Quantum Information, Lecture Notes in Physics, Vol. 587. Heidelberg-Berlin: Springer. Page numbers taken from arXiv:quant-ph/0010011.

Przibram, K. (ed.) (1967). Letters on Wave Mechanics: Schrödinger, Planck, Einstein, Lorentz. Translated from German and introduction by M. J. Klein. New York: Philosophical Library.

Rynasiewicz, R. (2015). “The (?) Correspondence Principle.” Pp. 175-199 in F. Aaserud and H. Kragh (eds.), One Hundred Years of the Bohr Atom: Proceedings from a Conference. (Scientia Danica: Series M: Mathematica et Physica, 1). Copenhagen: Royal Danish Academy of Sciences and Letters.

Schlosshauer, M. (2007). Decoherence and the Quantum-to-Classical Transition. Berlin: Springer.

Schrödinger, E. (1926). "Quatisierung als eigenwertproblem (Vierte mitteilung).” Annalen der Physik, 81: 437-490. English traslation in E. Schrödinger, Collected Papers on Wave Mechanics 1928. London and Glasgow: Blackie \& Son Limited.

Schuster, H. G. (1984). Deterministic Chaos. Weinheim: VCH.

Smith, P. (1998). Explaining Chaos. Cambridge: Cambridge University Press.

Sommerfeld, A. (1923). Atomic Structure and Spectral Lines, translated by H. Brose. London: Methuen (original 1919).

Sommerfeld, A. (1924). "Grundlagen der Quantentheorie und des Bohrschen Atommodelles." Die Naturwissenschaften, 12: 1047-1049.

van Hove, L. (1957). "The approach to equilibrium in quantum statistics." Physica, 23: 441480.

van Hove, L. (1959). “The ergodic behaviour of quantum many-body systems." Physica, 25: 268-276. 
van Kampen, N. (1954). "Quantum statistics of irreversible processes.” Physica, 20: 603-622.

van Vleck, J. (1924). "The absoption of radiation by multiply periodic orbits and its relation to the correspondence principle and the Rayleigh-Jeans law. Part 1. Some extensions of the correspondence principle." Physical Review, 24: 330-346.

Viola, L. and Barnum, H. (2010). "Entanglement and subsystems, entanglement beyond subsystems, and all that." Pp. 16-43 in A. Bokulich and G. Jaeger (eds.), Philosophy of Quantum Information and Entanglement. Cambridge: Cambridge University Press.

Yvon, J. (1935). La Théorie Statistique des Fluides et l'Équation d'État. Paris: Hermann.

Zanardi, P. (2001). "Virtual quantum subsystems." Physical Review Letters, 87: 077901.

Zeh, H.-D. (1970). "On the interpretation of measurement in quantum theory." Foundations of Physics, 1: 69-76.

Zeh, H.-D. (1973). "Toward a quantum theory of observation." Foundations of Physics, 3: 109-116.

Zeh, H.-D. (2005). "Roots and fruits of decoherence.” Séminaire Poincaré, 2: 1-19.

Zurek, W. (1981). "Pointer basis of quantum apparatus: into what mixtures does the wave packet collapse?" Physical Review D, 24: 1516-1525.

Zurek, W. (1982). "Environment-induced superselection rules.” Physical Review D, 26: 18621880.

Zurek, W. (1991). "Decoherence and the transition from quantum to classical." Physics Today, 44: 36-44.

Zurek, W. (1994). "Preferred sets of states, predictability, classicality and environmentinduced decoherence.” Pp. 175-207 in J. J. Halliwell, J. Pérez-Mercader, and W. H. Zurek (eds.), Physical Origins of Time Asymmetry. Cambridge: Cambridge University Press.

Zurek, W. (1998). "Decoherence, einselection, and the existential interpretation." Philosophical Transactions of the Royal Society A, 356: 1793-1820. Page numbers taken from arXiv:quant-ph/0010011.

Zurek, W. (2003). "Decoherence, einselection, and the quantum origins of the classical." Reviews of Modern Physics, 75: 715-776. Page numbers taken from arXiv:quantph/0105127. 